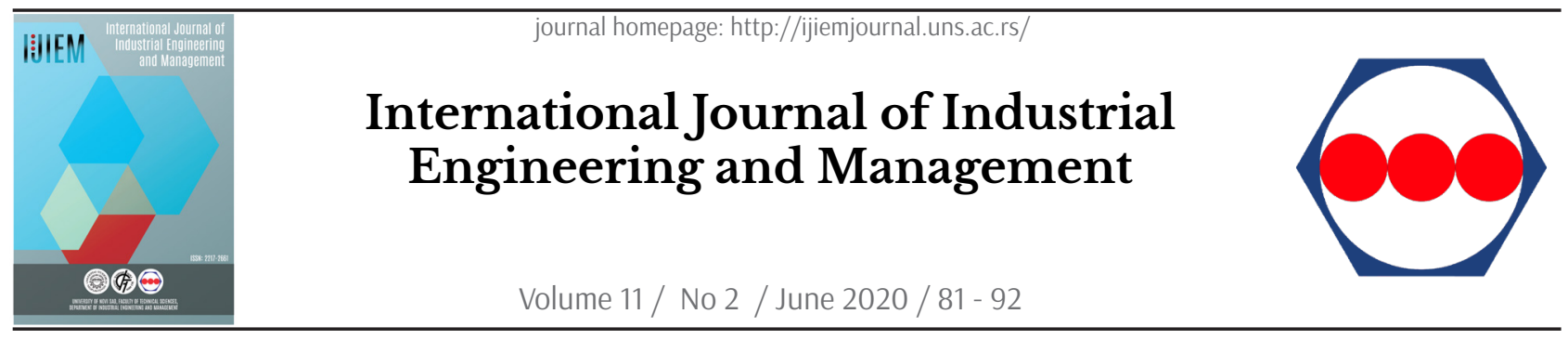

Original research article

\title{
OMCTA: A novel knowledge-based technique to evaluate the influence of $0 \& M$ actions on maintenance management
}

\author{
C.A. Murad ${ }^{a}$, A.H.A. Melani ${ }^{\text {a*}}$, M.A.C. Michalski ${ }^{a}$, A.C. Netto ${ }^{a}$, G.F.M. Souza $^{a}$, S.I. Nabeta $^{b}$ \\ a Department of Mechatronics and Mechanical Systems, University of São Paulo, Avenida Professor Mello de Moraes 2231, \\ São Paulo, SP, 05508-030, Brazil; \\ ${ }^{b}$ Department of Energy and Electrical Automation, University of São Paulo, Av. Prof. Luciano Gualberto, Travessa 3, 158, \\ São Paulo, SP, 05508-010, Brazil
}

\section{ABSTRACT}

Human error is a major cause of unavailability of industrial equipment. Consequently, several approaches attempt to assess how this issue influences maintenance management. However, these techniques are generally limited, not distinguishing between $O \& M$ actions. In this way, this work presents a new method, named OMCTA, based on techniques already known and widespread both in academia and industry. By structuring the expert knowledge, the method separately quantifies the likelihood of O\&M actions causing failures. A case study is analyzed, bringing results that clearly show the effects of the O\&M actions for different Kaplan turbine failure modes.

\section{ARTICLE INFO}

Article history:

Received September 25, 2019

Revised March 10, 2020

Accepted March 24, 2020

Published online April 14, 2020

Keywords:

Critical Systems;

Equipment O\&M;

Maintenance Management;

Expert Knowledge-Based Techniques

*Corresponding author:

Arthur Henrique de Andrade Melani melani@usp.br

\section{Introduction}

A proper maintenance management is undoubtedly crucial to achieve and maintain productivity, competitiveness, and profitability of companies in any industry. At first seen as the process for maintaining a company's assets and resources, maintenance management faces new challenges and needs to go much further in times of economic instability, aggressive electrification of energy end uses and the rise of industry 4.0. Anticipation becomes a key word, even in best case scenarios were a good maintenance planning is supported by modern maintenance philosophies such as Risk Based Maintenance (RBM), Reli- ability Centered Maintenance (RCM) and Condition Based Maintenance (CBM) [1].

The Operation and Maintenance (O\&M) costs in basic industries such as power generation, mining, or oil and gas constitute a major concern among managers, scientists, and engineers. Poorly operated and maintained machines can lead to random failures and, consequently, to production interruption.

Furthermore, poor O\&M always have an extra cost, and losses can range from a couple of thousand USD to immeasurable values, such as in cases where human lives can be affected or lost. Identifying the cause of poor O\&M allows the anticipation of causes of critical items failures, avoiding such significant 
losses.

Although some well proven techniques have been used to support maintenance management regarding human errors [2], as far as it could be detected in the existing literature, this subject is still generally approached in a traditional simplistic way, i.e., considering as separate effects the influences of operational actions and of human errors on equipment condition. The authors, however, consider that it is high time that the subject of plant maintenance management be studied using a new complex engineering approach capable of accurately distinguishing the respective influences of O\&M actions and then synthesizing both effects in a combined figure.

Therefore, in this paper a novel expert knowledge-based technique, the Operation and Maintenance Causal Tree Analysis (OMCTA), is presented. Designed to integrate in a simple graphical and numeric manner the combined effects of O\&M actions on equipment health and life expectancy, OMCTA may provide maintainers with a clearer view of what must be taken into consideration to increase as much as possible equipment availability.

OMCTA is based on disseminated and valued techniques. The idea is that experts can evaluate the basic O\&M activities broken down into components leading to failures, as is done in Causal Tree Analysis (CTA), while considering a logic structure with "AND" and "OR" gates similar to that used in Fault Tree Analysis (FTA). In this way, the method innovates by structuring expert knowledge through these techniques, quantifying the likelihood of O\&M actions causing failures.

\section{Literature review}

Throughout the last decades, the importance of the maintenance function, as well as its management, has increased considerably [3]. As organizations attempt to protect capital investments and generate peak efficiency, O\&M activities have become increasingly critical. Also, in order to adequately execute the maintenance plan a management strategy must be selected.

Barberá et al. [4] present an advanced model for the integrated management for industrial plant maintenance. The proposed model aligns the local maintenance objectives with the overall business objectives, aiming at achieving maintenance effectiveness. It is noteworthy that human resources management plays an important role to achieve maintenance goals. Staff motivation, preparation and training on specific tasks affect maintenance efficiency and equip- ment performance. In fact, committed leadership and maintenance oriented toward Total Productive Maintenance (TPM) were found to be the key factors to high performing power plants [5].

Although maintenance departments are not directly involved in production, they certainly have an important bearing on both quality and cost of the product, playing an important role on a company assurance of a sustainable future. The maintenance management goal is to bring down downtime and increase availability [6], consequently increasing profitability.

Even in product design the maintenance of database associated with product configuration plays an important role in providing industry profitability. Rasmussen et al. [7] discuss the importance of maintaining systems that deal with product information since incomplete or outdated information strongly affects product quality and customers' satisfaction.

Managing maintenance involves several activities such as: planning of preventive maintenance actions; scheduling of activities considering available resources and planned production; management of spare parts; analysis of data to reduce the occurrence of failures and to improve performance of the maintenance function [8].

Also, efficient equipment maintenance processes demand accurate equipment data collection, clearly defined tasks, work order management, inventory control and reporting. In fact, integration of maintenance processes with other organization activities has crucial importance to achieve profitability [9].

As manufacturing technology becomes more complex, maintenance requirements become stricter and workers must have high-level skills and in-depth knowledge of the equipment. In this way, it is of great importance to evaluate the current state of the maintenance area of organizations and drive actions to increase efficiency and effectiveness, providing the support necessary to reach the level required by the new challenges [10].

Effectiveness represents the company's overall satisfaction with the capacity and condition of its assets or the reduction in the total cost as a result of permanent production capacity. Effectiveness then focuses on correcting the process and achieving the necessary result. On the other hand, effectiveness is associated with the selection of the Key Performance Index (KPI) that represents the link between the company's strategic plan and maintenance activities, and the selection of the most critical equipment for asset operation management [11].

Maintenance efficiency deals with providing the 
best maintenance service within budget constraints. To achieve efficiency maintenance management must define the most suitable strategy for maintenance planning, avoiding failures in critical asset equipment. Modern maintenance philosophies, such as RCM and RBM, define what are the most appropriate maintenance actions to be performed on critical equipment to prevent failures occurrence. These philosophies prioritize the application of predictive and preventive maintenance practices, preventing failures that require immediate corrective actions, increasing maintenance costs and production loss [12].

Nevertheless, those maintenance philosophies only consider the technical aspects associated with the equipment failure modes, i.e., considering that maintenance actions will prevent the occurrence of failure modes that affect the performance of critical parts of the equipment. The proposed maintenance plan assumes that the maintainers' technical skills, proper job preparation and tools are adequate to perform the tasks associated with the maintenance plan.

Thus, foreseeing the very cause of the fault condition, even anticipating the failure, should be considered as a target of maintenance planning. Excluding the expected wear-and-tear, operating and maintenance errors become the major cause of systems failures [2]. If not prepared, industrial plants would face human errors as maintainers perform their tasks [1315]. Thus, identifying and quantifying human errors are important assignments in the process of reviewing and correcting maintenance actions [16, 17].

Considering the Maintenance Resource Management (MRM) concept, Taylor [18] reveals a deep concern with the consequences of human factor occurrences in maintenance operations and Siddiqui, Iqbal and Manarvi [19] present an approach for the relevance of dealing timely with human factor occurrences that may lead to irreparable losses to men and equipment.

Alaswad and Xiang [20] mentioned that only a few CBM studies have investigated human error in CBM modelling, showing the need of integrating human reliability into CBM optimization models and investigating the effectiveness of resulting strategies in presence of human interferences.

In this regard, several methods for performing human reliability and error analysis can be found in the literature, such as Failure Mode and Effect Analysis (FMEA), Root Cause Analysis (RCA), FTA, Technics of Operation Review (TOR), Cause and Effect Diagram (CAED), Pareto Analysis, Hazard and Operability Study (HAZOP), Probability Tree Method, Pontecorvo Method, Markov Method, among others
$[21,22]$.

Among these techniques, FTA has been very popular for quite some time now. It is one of the most important logic and probabilistic techniques used in Probabilistic Risk Assessment (PRA) and system reliability assessment today. In fact, FTA and Causal Tree Analysis (CTA) are two very well-known and popular methods to analyze the risks related to safety and critical assets.

Ortmaier and Schellhorn [23] point out practical advantages of FTA used for safety application. Compared to other formal safety analysis methods, FTA is the one which has a human readable and understandable logic background structure and is easily accepted in industry.

Melani et al. [24] used the traditional FTA as a complement to HAZOP and Failure Mode, Effects and Criticality Analysis (FMECA) in the criticality determination process when analyzing measures for ensuring continuous equipment operation. Ferdous et al. [25] considered a systematic method for evaluating likelihood, consequences, and risk of adverse events by applying a fuzzy set theory and evidence theory approach to improve Quantitative Risk Analysis results when using Event Tree Analysis (ETA) and FTA.

Vesely and Stamatelatos [26] presented FTA as a deductive analysis approach for resolving an undesired event into its causes, or a backward-looking analysis of the causes of a given event, and called attention to a specific stepwise logic that was used.

Ruijters et al. [27] suggest Fault Maintenance Trees, connecting traditional FTA with maintenance strategies. The main idea was to convert the equipment degradation into the fault trees in addition to different inspection routines. Sarkar et al. [28] use FTA on a gas turbine to define and enumerate specifically the causes of the system failure modes in terms of equipment failures and human errors.

On the other hand, CTA is a powerful technique that shows the chain of events leading to a fault or even a failure. The idea is that incidents and accidents result from process variation. Vasconcelos, Senne and Marques evaluate the use of CTA method in investigation of incidents and accidents involving radioactive materials [29].

CTA displays in a logical and hierarchically structured way all the actions and conditions necessary and sufficient for a given consequence to have occurred, i.e. it provides a method of analyzing the critical human errors and technical failures that have contributed to an incident or accident in order to determine the root causes. This graphical technique is simple 
to perform and very flexible, mapping out the facts rather than being constrained to accident causation models.

Originally conceived for investigating the causes of incidents and accidents, only branches leading to the incident are represented in CTA. However, unlike FTA, no "OR gates" are used [29]. This considerable limitation precludes the use of CTA proper when dealing with maintenance related problems, requiring further developments.

Brik and ben Ammar [30], e.g., present an approach using analysis tools of reliability to describe the various phenomena causing the capacity deficiency of lead acid battery based on CTA (introducing, however, "OR gates" on the tree structure) to describe the fault origin and FTA to study the fault degradation.

Based on the bibliographic review, the need to ensure the efficiency and effectiveness of maintenance becomes an essential task for maintenance management. Thus, preventing the causes of failures, such as human error, is a natural and necessary path. Several tools are already used to assist in this task, such as FTA. However, none of these approaches clearly distinguish the effects of O\&M activities in the cause of failures. In addition, the need of "OR gates" in the tree structure seems clear, leading the authors of this work to propose the methodology presented in the next section.

\section{Methodology}

Despite their proven value, FTA and CTA are not capable of providing a clear distinction between the effects of inadequate O\&M practices upon maintenance requirements. They do not offer an entirely convenient distinction between the many aspects involved in the production of failures, including human error, flawed O\&M procedures, incorrect materials, and working environment constraints.
The proposed method offers a new expert knowledge-based procedure for analyzing potential O\&M errors during the execution of daily maintenance activities. Thus, plant O\&M teams could be able to evaluate potential risks, and to prioritize actions regarding equipment failure likelihood according to the results provided by the method.

The method development is divided into four steps, as shown in Fig. 1.

Firstly, the system is studied to obtain a good knowledge of it, understanding how it works, and how it is organized. Such specific knowledge can be obtained from the participation of specialists in the method application, interviews and questionnaires answered by $\mathrm{O} \& \mathrm{M}$ teams, and documentation and drawings of the analyzed system. Secondly, system is broken down into subsystems and items, which results in a system view like an FTA. Fig. 2 shows that the analysis starts with a system failure at the top of the tree - the top event - and works downwards through the leaves with the help of logic gates until the root causes of the failure are reached at the bottom. The analysis can be carried out both quantitatively using the probabilities of the several events, and qualitatively by means of minimal cut sets [15, 31].

The third step is the addition of the O\&M activities under each basic event. Then each activity component is scored according to the ranking criteria. It is considered that any incident such as a failure results from changes or variations in the normal process, i.e., in the states or usual conditions of any com-

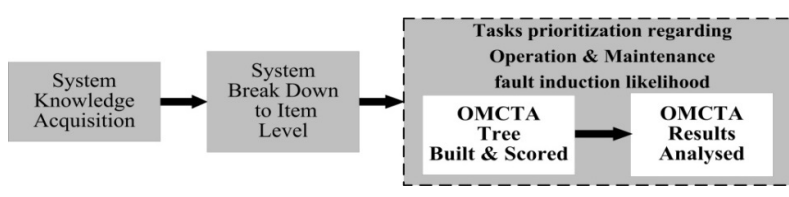

Figure 1. Proposed methodology

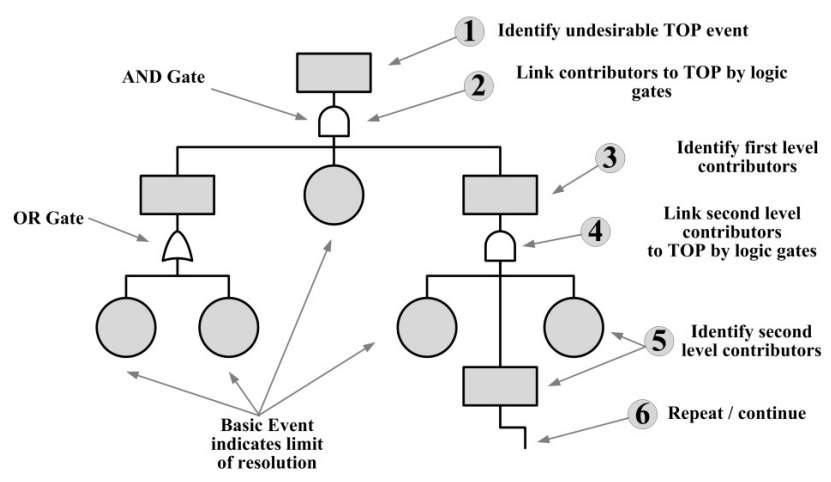

Figure 2. Basic structure of a Fault Tree 
ponent of an activity. Therefore, the changes in the system must be identified, listed, and structured as a diagram. The basic components of an activity leading to failures are listed on Fig. 3.

Finally, the results are analyzed and prioritized according to its scores. In this work, two ways of analysis are proposed: the direct comparison of the results obtained for the maintenance and operation weights for each failure mode; and a global analysis carried out from a prioritization matrix, showing how OMCTA can assist in decision making. Based on risk matrices, the prioritization matrix classifies the failures in terms of the need for prioritization of maintenance planning and the need for allocation of qualified labor.

\section{The proposed method}

Basically, OMCTA retains the basic characteristics of CTA, which is the breaking down of activities, in a logical structure with "AND" and "OR" gates, based on FTA. As a result, the structure of an OMCTA diagram has unique characteristics, as presented in Fig. 4.

OMCTA decomposes the failure of a system and its subsystems into items' failures, were the term "item" describes any piece of equipment that belongs to a subsystem. However, unlike a fault tree, operation (OPER) and maintenance (MAINT) of each item are considered as activities and subdivided into four components: individual (I), task (T), material (M), and work medium (WM). The combination of FTA and CTA basic characteristics can provide a greater flexibility for a more comprehensive analysis of both O\&M aspects.

This expanded combination of features provides a method of ranking items in relation to their likelihood of causing system failures that would be originated by either operational or maintenance actions. Then, the experts can grade each activity component according to the ranking criteria used for the numbers in the lower half of each component in Fig. 4, as presented in Table 1.

The ranking criteria for component INDIVIDUAL (I) considers the degree of expertise and experience required from individuals to perform O\&M

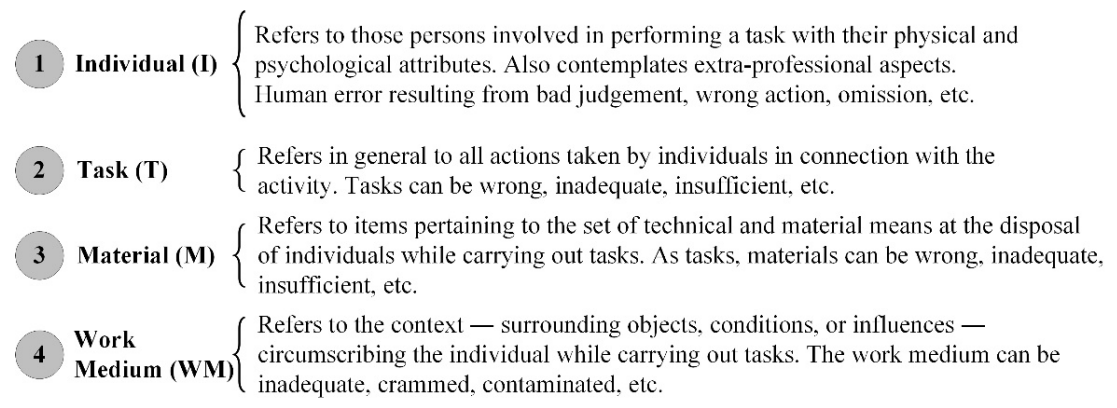

Figure 3. Components of an Activity

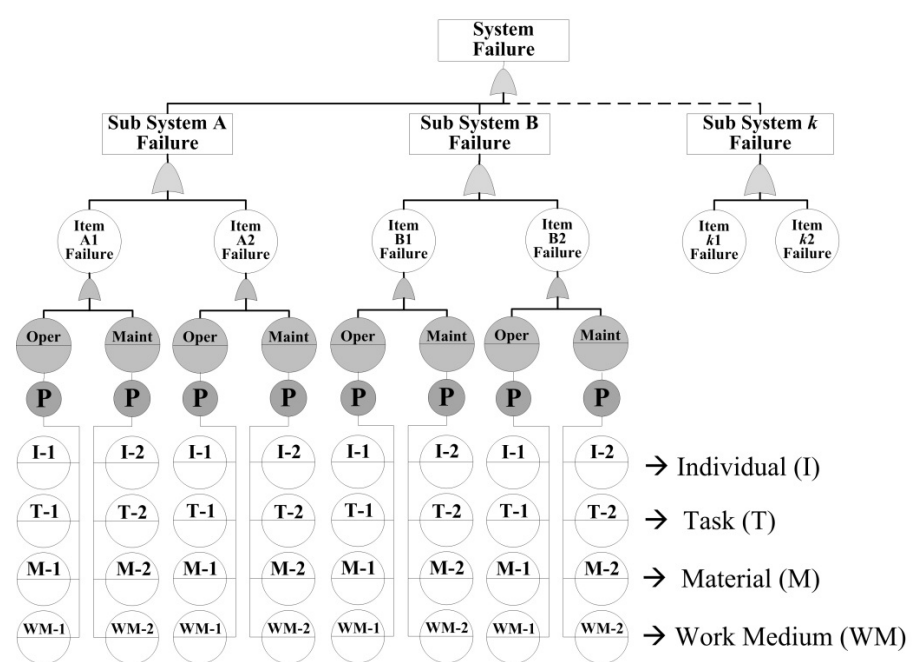

Figure 4. Operation \& Maintenance Causal Tree Analysis (OMCTA) 
Table 1. Ranking criteria for OMCTA components

\begin{tabular}{|c|c|c|c|}
\hline & $\mathrm{R}$ & Operation & Maintenance \\
\hline \multirow{5}{*}{ 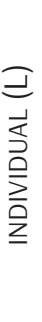 } & 1 & Requires low expertise and experience & Requires low expertise and experience \\
\hline & 2 & Requires low expertise and medium experience & Requires low expertise and medium experience \\
\hline & 3 & Requires medium expertise and medium experience & Requires medium expertise and medium experience \\
\hline & 4 & Requires medium expertise and high experience & Requires medium expertise and high experience \\
\hline & 5 & Requires high expertise and high experience & Requires high expertise and high experience \\
\hline \multirow{5}{*}{$\begin{array}{l}E \\
\frac{1}{v} \\
E\end{array}$} & 1 & Sporadic and normal working conditions & Low complexity and short duration \\
\hline & 2 & Intermittent and normal working conditions & Medium complexity and short duration \\
\hline & 3 & Continuous and normal working conditions & Medium complexity and medium duration \\
\hline & 4 & Intermittent / strenuous working conditions & High complexity and medium duration \\
\hline & 5 & Continuous / strenuous working conditions & High complexity and long duration \\
\hline \multirow{5}{*}{ 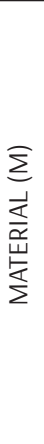 } & 1 & Requires few resources with high availability & $\begin{array}{l}\text { Requires resources with high availability and ordinary } \\
\text { tools }\end{array}$ \\
\hline & 2 & Requires many resources with high availability & $\begin{array}{l}\text { Requires resources with high availability and specialized } \\
\text { tools }\end{array}$ \\
\hline & 3 & Requires few resources with medium availability & $\begin{array}{l}\text { Requires resources with medium availability and special- } \\
\text { ized tools }\end{array}$ \\
\hline & 4 & Requires many resources with medium availability & $\begin{array}{l}\text { Requires resources with medium availability and com- } \\
\text { plex tools or Requires resources with low availability } \\
\text { and specialized tools }\end{array}$ \\
\hline & 5 & Requires resources with low availability & $\begin{array}{l}\text { Requires resources with low availability and complex } \\
\text { tools }\end{array}$ \\
\hline \multirow{5}{*}{ 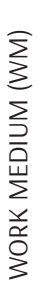 } & 1 & $\begin{array}{l}\text { No external interferences such as vibration, magnetic field, } \\
\text { high temperature, high humidity, air contamination, etc. }\end{array}$ & Clean air, non-confined space, and easy access \\
\hline & 2 & One external interference of low magnitude & Clean air, non-confined space, and difficult access \\
\hline & 3 & More than one external interference of low magnitude & Clean air and confined space \\
\hline & 4 & At least one external interference of medium magnitude & Contaminated air and non-confined space \\
\hline & 5 & At least one external interference of high magnitude & Contaminated air and confined space \\
\hline
\end{tabular}

tasks with several possible combinations.

Ranking criteria for component TASK (T) show the essential differences between the respective effects of $O \& M$ tasks on equipment life and performance, as revealed by failures and their consequences. While frequency and demand seem to adequately characterize the impact of operational tasks, complexity and duration provide a better assessment of the influence of maintenance tasks.

In the ranking criteria for component MATERIAL (M), the term "resources" is meant to include both human and material resources of any kind, including hardware, software, processed materials, and information, whose unavailability may be detrimental to the operation performance.

The ranking criteria for component WORK MEDIUM (WM) consider the unfavorable influence of external aspects on O\&M tasks. In addition, on the maintenance side the term "confined" implies the idea of reduced mobility due to any cause, cramming included.

Although basically built on the tradition of treeshaped techniques, OMCTA derives its analytical essence from two major disciplines: strategy and logistics. In other words, with timely acquiring, receiving, and preparing all the human and material resources needed to take a vantage point before the moment of action.

To demonstrate the application of OMCTA in a real situation, a case study considering a Kaplan turbine will be presented in the next section.

\section{Case study}

To illustrate the use of OMCTA, the technique was applied considering data from a Brazilian run-ofthe-river baseload Hydroelectric Power Plant (HPP) located in the North region of the country. The plant has three generating units with Kaplan turbines and an installed capacity of approximately 450 MW. 
As a premise for the system analysis, it was considered that the units' operation occurs in ideal conditions, with dispatch close to the best efficiency design point defined by the hydro generators' hill chart [32]. In this condition, any equipment breakdown during steady state operation will reduce the generation output and system availability.

\subsection{Development}

The analyzed equipment is extremely important for the application of OMCTA, since in the item failure identification phase one needs to enumerate all potential failure factors that affect equipment availability.

Experts could first investigate FMEA to find out which failure modes were involved and examine relevant information from past records. Then apply their knowledge to list potential failure modes and afterwards use OMCTA to identify how O\&M actions could affect each one. The OMCTA model is concerned with expertise and experience of the individual, the working conditions, the complexity and duration of the task. In addition, it takes into con- sideration the availability of resources such as spare parts and special tools needed for the task. Finally, it considers external interferences and the work place during task completion. After having displayed all the subsystems and constituent items, the experts can score each one of the four components for the O\&M activities.

Fig. 5 shows part of the developed OMCTA of a Kaplan turbine and Table 2 shows the OMCTA ranking for O\&M activities considering the failure modes and failure root causes identification for a Kaplan turbine. Some item failures appear in different rows of Table 2 for different failure modes with the same OMCTA results. These are common cause failures and it is important to note that they can be particularly meaningful due to this redundancy of similar items subject to the same O\&M actions. Disregarding the common cause failures replication, which results in 26 different item failures, and sorting from the greatest to the lowest likelihood of causing failures due O\&M actions, the respective OMCTA rankings are presented in Fig. 6 .

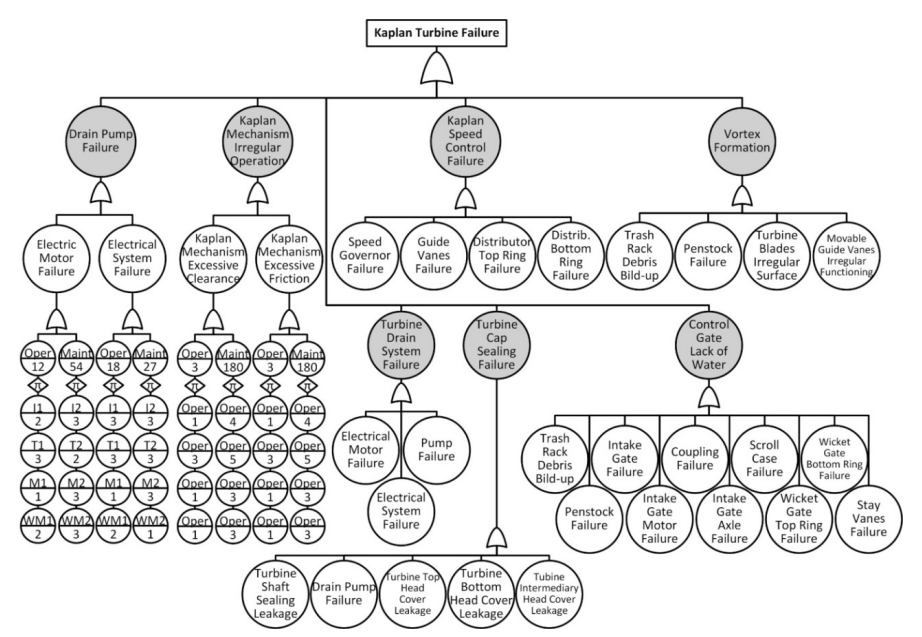

Figure 5. Failure modes identification of a Kaplan turbine
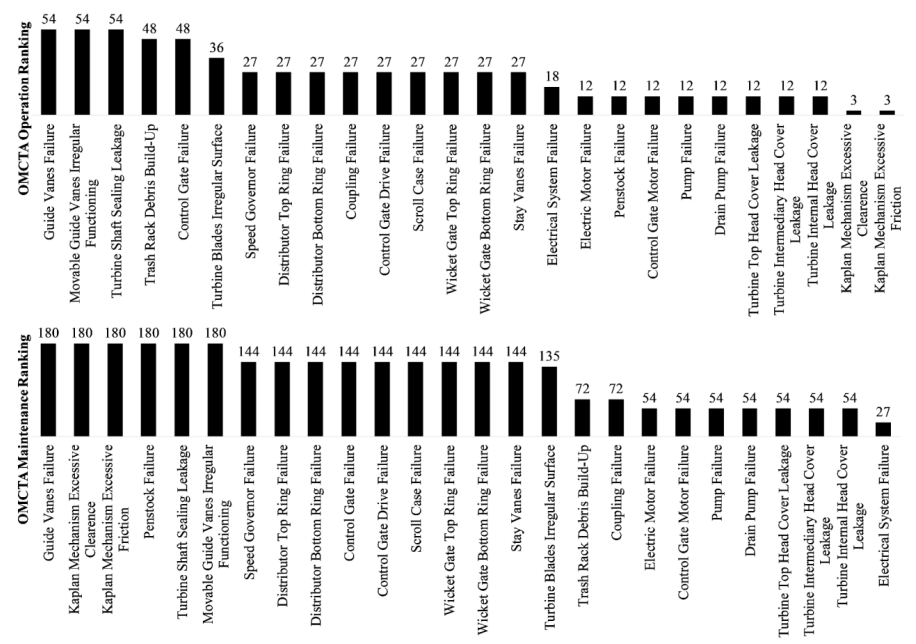

Figure 6. OMCTA operation and maintenance rankings 
Table 2. OMCTA ranking for Operation and Maintenance

\begin{tabular}{|c|c|c|c|}
\hline Subsystem Failure & Item Failure & OPER & MAINT \\
\hline \multirow{3}{*}{ Drain Pump Failure } & Electric Motor Failure & 12 & 54 \\
\hline & & & \\
\hline & Electric Motor Failure & 18 & 27 \\
\hline \multirow{4}{*}{ Kaplan Speed Control Failure } & Speed Governor Failure & 27 & 144 \\
\hline & Guide Vanes Failure & 54 & 180 \\
\hline & Distributor Top Ring Failure & 27 & 144 \\
\hline & Distributor Bottom Ring Failure & 27 & 144 \\
\hline \multirow{2}{*}{ Kaplan Mechanism Irregular Operation } & Kaplan Mechanism Excessive Clearance & 3 & 180 \\
\hline & Kaplan Mechanism Excessive Friction & 3 & 180 \\
\hline \multirow{4}{*}{ Drain Pump Failure Vortex Formation } & Trash Rack Debris Build-Up & 48 & 72 \\
\hline & Penstock Failure & 12 & 180 \\
\hline & Turbine Blades Irregular Surface & 36 & 135 \\
\hline & Movable Guide Vanes Irregular Functioning & 54 & 180 \\
\hline \multirow{10}{*}{ Intake Lack of Water } & Trash Rack Debris Build-Up & 48 & 72 \\
\hline & Penstock Failure & 12 & 180 \\
\hline & Control Gate Failure & 48 & 144 \\
\hline & Control Gate Motor Failure & 12 & 54 \\
\hline & Coupling Failure & 27 & 72 \\
\hline & Control Gate Drive Failure & 27 & 144 \\
\hline & Scroll Case Failure & 27 & 144 \\
\hline & Wicket Gate Top Ring Failure & 27 & 144 \\
\hline & Wicket Gate Bottom Ring Failure & 27 & 144 \\
\hline & Stay Vanes Failure & 27 & 144 \\
\hline \multirow{3}{*}{ Turbine Drain System Failure } & Electric Motor Failure & 12 & 54 \\
\hline & Pump Failure & 12 & 54 \\
\hline & Electrical System Failure & 18 & 27 \\
\hline \multirow{5}{*}{ Turbine Cap Sealing Failure } & Turbine Shaft Sealing Leakage & 54 & 180 \\
\hline & Drain Pump Failure & 12 & 54 \\
\hline & Turbine Top Head Cover Leakage & 12 & 54 \\
\hline & Turbine Intermediary Head Cover Leakage & 12 & 54 \\
\hline & Turbine Internal Head Cover Leakage & 12 & 54 \\
\hline
\end{tabular}

\subsection{Analysis}

Analyzing the OMCTA results from Fig. 6, it is clear that maintenance bearing on the likelihood of causing failures is greater than operation. Thus, a major conclusion can be reached: in addition to the expected wear-and-tear, for a Kaplan turbine, present maintenance actions may be the major cause of future maintenance.

It is important to highlight that in this work, fail- ures related to the installation and assembly during hydrogenerator erection are not considered, since they would be mitigated during the machine commissioning. On the other hand, during the turbine life cycle, assemblies and installations will be considered as maintenance activities, falling into only two sources of failure (in addition to natural wear), contrary to what is presented by Dhillon [2], who considers failures related to operation, maintenance, assembly and installation throughout the life cycle of the equip- 
Table 3. OMCTA Operation and Maintenance weights

\begin{tabular}{|c|c|c|c|c|c|}
\hline Item Failure & OPER Weight & MAINT Weight & Total Weight & $\%$ OPER & $\%$ MAINT \\
\hline Trash Rack Debris Build-Up & 48 & 72 & 120 & $40.0 \%$ & $60.0 \%$ \\
\hline Electrical System Failure & 18 & 27 & 45 & $40.0 \%$ & $60.0 \%$ \\
\hline Movable Guide Vanes Irregular Functioning & 54 & 180 & 162 & $33.3 \%$ & $66.7 \%$ \\
\hline Coupling Failure & 27 & 72 & 99 & $27.3 \%$ & $72.7 \%$ \\
\hline Control Gate Failure & 48 & 144 & 192 & $25.0 \%$ & $75.0 \%$ \\
\hline Guide Vanes Failure & 54 & 180 & 234 & $23.1 \%$ & $76.9 \%$ \\
\hline Turbine Shaft Sealing Leakage & 54 & 180 & 234 & $23.1 \%$ & $76.9 \%$ \\
\hline Turbine Blades Irregular Surface & 36 & 135 & 171 & $21.1 \%$ & $78.9 \%$ \\
\hline Control Gate Motor Failure & 12 & 54 & 66 & $18.2 \%$ & $81.8 \%$ \\
\hline Drain Pump Failure & 12 & 54 & 66 & $18.2 \%$ & $81.8 \%$ \\
\hline Electric Motor Failure & 12 & 54 & 66 & $18.2 \%$ & $81.8 \%$ \\
\hline Pump Failure & 12 & 54 & 66 & $18.2 \%$ & $81.8 \%$ \\
\hline Turbine Intermediary Head Cover Leakage & 12 & 54 & 66 & $18.2 \%$ & $81.8 \%$ \\
\hline Turbine Internal Head Cover Leakage & 12 & 54 & 66 & $18.2 \%$ & $81.8 \%$ \\
\hline Turbine Top Head Cover Leakage & 12 & 54 & 66 & $18.2 \%$ & $81.8 \%$ \\
\hline Control Gate Drive Failure & 27 & 144 & 171 & $15.8 \%$ & $84.2 \%$ \\
\hline Distributor Bottom Ring Failure & 27 & 144 & 171 & $15.8 \%$ & $84.2 \%$ \\
\hline Distributor Top Ring Failure & 27 & 144 & 171 & $15.8 \%$ & $84.2 \%$ \\
\hline Scroll Case Failure & 27 & 144 & 171 & $15.8 \%$ & $84.2 \%$ \\
\hline Speed Governor Failure & 27 & 144 & 171 & $15.8 \%$ & $84.2 \%$ \\
\hline Stay Vanes Failure & 27 & 144 & 171 & $15.8 \%$ & $84.2 \%$ \\
\hline Wicket Gate Bottom Ring Failure & 27 & 144 & 171 & $15.8 \%$ & $84.2 \%$ \\
\hline Wicket Gate Top Ring Failure & 27 & 144 & 171 & $15.8 \%$ & $84.2 \%$ \\
\hline Penstock Failure & 12 & 180 & 192 & $6.3 \%$ & $93.8 \%$ \\
\hline Kaplan Mechanism Excessive Clearance & 3 & 180 & 183 & $1.6 \%$ & $98.4 \%$ \\
\hline Kaplan Mechanism Excessive Friction & 3 & 180 & 183 & $1.6 \%$ & $98.4 \%$ \\
\hline
\end{tabular}

ment.

Therefore, avoiding unnecessary maintenance is not only a matter of reducing predictable machine downtime, but also avoiding future unforeseen failures. On the other hand, the likelihood for the same item failures can vary significantly considering the influence of O\&M activities. Table 3 presents, in descending order of operation weight, the OMCTA ranking considering an overall value, resulting from the sum of the operation and maintenance values, and the maintenance and operation percentual values for each item failure. The average value found for the operation percentual weight is $18.7 \%$, while for the maintenance is $81.3 \%$. These results support the idea that the likelihood of maintenance actions causing equipment failures is greater than that of operation actions.

At the bottom of Table 3, the presented values show that under normal conditions, mainly maintenance activities can cause equipment failures in addition to the normally expected wear-and-tear. The root causes of Kaplan mechanism failure are related to the assembly and lubrication thereof, both maintenance activities. In the first case, an incorrectly assembly could cause excessive clearance between items, torque specification either too low or too high. In the second case, poor lubrication would result in friction and premature wear of the items, consequently causing excessive clearance. In these cases, maintenance planning becomes even more relevant, since a poorly done or unnecessary intervention may become the cause of a problem that previously did not exist.

Top items on Table 3 show a balance between the impact likelihood of both maintenance and operation actions in the item failure. In these cases, working conditions for maintenance and operation are quite similar. Considering trash rack debris buildup as an example, the weights have close values. In this case, maintenance activities are simpler, since the cleaning of the trash rack can be automated, reducing the risk of human failures during such actions, while the bearing of operation becomes higher due to the existence of external factors that influence the activity's results (such as the amount of debris in the river). 


\subsection{Decision making via OMCTA}

The analysis performed in the previous example demonstrates how the results of OMCTA relate to the likelihood of O\&M actions causing item failures. However, despite the great potential already presented, OMCTA can be very useful as a powerful tool to support decision-making in reliability engineering, i.e., regarding maintenance planning and the allocation of qualified labor for certain activities. The method can be useful in a scenario where the technical staff is reduced, a usual situation in hydroelectric power plants. To allow decision-making visually easier with the results of the OMCTA, it is possible to use a prioritization matrix, as Table 4 shows.

Once having the maintenance and operation like-

Table 4. Prioritization matrix

Maintenance Score

1 to $50 \quad 51$ to $100 \quad 101$ to $150 \quad 151$ to 200

\begin{tabular}{|c|c|c|c|c|c|}
\hline \multirow{4}{*}{ 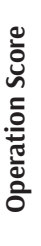 } & 1 to 50 & Low & Low & Medium & High \\
\hline & 51 to 100 & Low & Medium & Medium & High \\
\hline & 101 to 150 & Medium & Medium & High & High \\
\hline & 151 to 200 & High & High & High & High \\
\hline
\end{tabular}

Table 5. Item failure classification according to the prioritization matrix

\begin{tabular}{|c|c|c|c|}
\hline Item Failure & OPER & MAINT & Prioritization \\
\hline Guide Vanes Failure & 54 & 180 & High \\
\hline Kaplan Mechanism Excessive Clearance & 3 & 180 & High \\
\hline Kaplan Mechanism Excessive Friction & 3 & 180 & High \\
\hline Penstock Failure & 12 & 180 & High \\
\hline Penstock Failure & 12 & 180 & High \\
\hline Turbine Shaft Sealing Leakage & 54 & 180 & High \\
\hline Speed Governor Failure & 27 & 144 & Medium \\
\hline Distributor Top Ring Failure & 27 & 144 & Medium \\
\hline Distributor Bottom Ring Failure & 27 & 144 & Medium \\
\hline Turbine Blades Irregular Surface & 36 & 135 & Medium \\
\hline Movable Guide Vanes Irregular Functioning & 54 & 108 & Medium \\
\hline Control Gate Failure & 48 & 144 & Medium \\
\hline Control Gate Drive Failure & 27 & 144 & Medium \\
\hline Scroll Case Failure & 27 & 144 & Medium \\
\hline Wicket Gate Top Ring Failure & 27 & 144 & Medium \\
\hline Wicket Gate Bottom Ring Failure & 27 & 144 & Medium \\
\hline Stay Vanes Failure & 27 & 144 & Medium \\
\hline Electric Motor Failure & 12 & 54 & Low \\
\hline Electrical System Failure & 18 & 27 & Low \\
\hline Trash Rack Debris Bild-Up & 48 & 72 & Low \\
\hline Trash Rack Debris Build-Up & 48 & 72 & Low \\
\hline Control Gate Motor Failure & 12 & 54 & Low \\
\hline Coupling Failure & 27 & 72 & Low \\
\hline Electric Motor Failure & 12 & 54 & Low \\
\hline Pump Failure & 12 & 54 & Low \\
\hline Electrical System Failure & 18 & 27 & Low \\
\hline Drain Pump Failure & 12 & 54 & Low \\
\hline Turbine Top Head Cover Leakage & 12 & 54 & Low \\
\hline Turbine Intermediary Head Cover Leakage & 12 & 54 & Low \\
\hline Turbine Internal Head Cover Leakage & 12 & 54 & Low \\
\hline
\end{tabular}


lihood of causing item failures obtained from OMCTA, a given fault is classified into one of three possible prioritization levels: High, Medium, and Low. It is important to note that the scales used in the development of the matrix must respect the maximum values of O\&M weights obtained with OMCTA. In Table 3, for example, the maintenance scale varies from 1 to 180, while the operating scale varies from 1 to 54 .

The way in which such matrix is assembled depends on how the company intends to use ranking in the prioritizing process and thus can present different configurations. Table 5 presents the classification of the item failures in the case study according to the prioritization matrix. This classification highlights the need to prioritize maintenance planning and the allocation of skilled labor to operate and maintain plant items.

\section{Discussion and conclusions}

In this paper a novel expert knowledge-based technique is presented, integrating in a simple graphical and numeric manner the combined effects of O\&M actions on equipment health and life expectancy. The combination of FTA and CTA basic characteristics provides OMCTA with a much greater flexibility and a more comprehensive analysis of O\&M aspects, implying in more suitable weighted means of discriminating the difference between influences of O\&M actions on maintenance planning. In this way, OMCTA may provide maintainers with a clearer view of what must be taken into consideration to increase as much as possible equipment availability by structuring expert knowledge and quantifying, separately, the likelihood of O\&M actions causing failures.

The results of OMCTA can be analyzed both from the point of view of O\&M actions, separately, verifying for each case which item failure is more significant (Fig. 6), and from the point of view of each item failure, verifying the influence of O\&M in each case (Table 3). Furthermore, from the prioritization matrix proposed in this work, item failures can be classified (Table 5), allowing decision-making visually easier through the results of the OMCTA.

With this range of possibilities, OMCTA allows the maintainer to define the maintenance actions priority as well as the manager to define in which aspects the operation and maintenance need to be improved, bringing implications for the control of spare parts, planning of activities, training of the teams, among other important aspects.
However, it should be noted that the method still has some restrictions. First, like any knowledge-based method, OMCTA is subject to the influence of epistemic uncertainties. In addition, the method is based on the premise that the analyzed system is commissioned and, thus, the origin of the failures due to human error would be restricted only to O\&M actions. Moreover, it is well known that methods and techniques not conducive to automatization, no matter how good they may look, have little chance of widespread practical application nowadays.

Therefore, the authors are already considering the automatization of the presented method as a subject for future research, as well as the incorporation of techniques that will contribute to the reduction or evaluation of the uncertainty level of the method.

\section{Funding}

This study was financed in part by the Coordenação de Aperfeiçoamento de Pessoal de Nível Superior - Brasil (CAPES) - Finance Code 001. The authors thank the financial support of FDTE, CAPES and EDP for the development of the present research.

\section{References}

[1] G. Niu, B. S. Yang, and M. Pecht, "Development of an optimized condition-based maintenance system by data fusion and reliability-centered maintenance," Reliab. Eng. Syst. Saf., vol. 95, no. 7, pp. 786-796, 2010.

[2] B. S. Dhillon, "Human error in maintenance: An investigative study for the factories of the future," IOP Conference Series: Materials Science and Engineering, vol. 65, 27th International Conference on CADCAM, Robotics and Factories of the Future 2014, London, UK, 22-24 Jul. 2014.

[3] R. Dekker, "Applications of maintenance optimization models: a review and analysis," Reliab. Eng. Syst. Saf., vol. 51, no. 3, pp. 229-240, Mar. 1996.

[4] L. Barberá, A. Crespo, and P. Viveros, "Advanced model for maintenance management in a continuous improvement cycle: integration into the business strategy," Int. J. Syst. Assur. Eng. Manag., vol. 3, no. 1, pp. 47-63, Jan.-Mar. 2012.

[5] S. Wai Foon and M. Terziovski, "The impact of operations and maintenance practices on power plant performance," J. Manuf. Technol. Manag., vol. 25, no. 8, pp. 1148-1173, Sep. 2014.

[6] J. Thomas, "A History of Maintenance: Implementing MP2," Honors College Capstone Experience/Thesis Projects, 2007.

[7] J. B. Rasmussen, A. Myrodia, L. Hvam, and N. H. Mortensen, "Cost of Not Maintaing a Product Configuration System", International Journal of Industrial Engineering and Management (IJIEM), vol. 9 no. 4, pp. 205-214, 2018.

[8] I. Lopes, P. Senra, S. Vilarinho, V. Sá, C. Teixeira, J. Lopes, A. Alves, J. A. Oliveira, M. Figueiredo, "Requirements 
specification of a computerized maintenance management system - a case study", Procedia CIRP, vol. 52, pp. 268-273, 2016.

[9] B. Stevanov, N. Zuber, R. Šostakov, Z. Tešić, S. Bojić, M. Georgijević, and A. Zelić, "Reengineering the Port Equipment Maintenance Process," International Journal of Industrial Engineering and Management (IJIEM), vol. 7 no. 3, pp. 103-109, 2016.

[10] M. A. Oliveira and I. Lopes, "Evaluation and improvement of maintenance management performance using a maturity model," International Journal of Productivity and Performance Management (IJPPM), vol. ahead-of-print no. ahead-of-print, Aug. 2019.

[11] A. C. Márquez, P. M. de León, J. F. G. Fernández, C. P. Márquez, and V. González, The maintenance management framework: A practical view to maintenance management, Safety, Reliability and Risk Analysis: Theory, Methods and Applications - Martorell et al. (eds), Taylor \& Francis Group, London, 2009.

[12] M. Braglia, D. Castellano, and M. Gallo, "A novel operational approach to equipment maintenance: TPM and RCM jointly at work," Journal of Quality in Maintenance Engineering (JQME), vol. 25, no. 4, pp. 612-634, 2019.

[13] P. Okoh and S. Haugen, "Maintenance-related major accidents: Classification of causes and case study," J. Loss Prev. Process Ind., vol. 26, no. 6, pp. 1060-1070, Nov. 2013.

[14] E. Bowen, "Predicting Impact of Maintenance Resource Management Training Utilizing Individual Difference Variables,"J. Aviat. Educ. Res., vol. 22, no. 3, 2013.

[15] M. Milczarek and J. Kosk-Bienko, Maintenance and Occupational Safety and Health: A statistical picture. Luxembourg: European Agency for Safety and Health at Work, 2010.

[16] I. Morag, P. Chemweno, L. Pintelon, and M. Sheikhalishahi, "Identifying the causes of human error in maintenance work in developing countries," Int. J. Ind. Ergon., vol. 68, no. August, pp. 222-230, Nov. 2018.

[17] M. J. Carr and A. H. Christer, "Incorporating the potential for human error in maintenance models," J. Oper. Res. Soc., vol. 54, no. 12, pp. 1249-1253, Dec. 2003.

[18]J. C. Taylor, "Evaluating the Effects of Maintenance Resource Management (MRM) Interventions in Airline Safety," Washington, DC, 1998.

[19] M. H. Siddiqui, A. Iqbal, and I. A. Manarvi, "Maintenance Resource Management: A key process initiative to reduce human factors in aviation maintenance," in 2012 IEEE Aerospace Conference, pp. 1-7, 2012.

[20] S. Alaswad and Y. Xiang, "A review on condition-based maintenance optimization models for stochastically deteriorating system," Reliab. Eng. Syst. Saf., vol. 157, pp. 54-63, Jan. 2017.

[21] B. S. Dhillon, Human reliability and error in transportation systems, Springer series in reliability engineering, Springer-Verlag, London, 2007.

[22] B. S. Dhillon, "Methods for performing human reliability and error analysis in health care," International Journal of Health Care Quality Assureance, vol. 16, no. 6, pp. 306-317, 2003.

[23] F. Ortmeier and G. Schellhorn, "Formal Fault Tree Analysis - Practical Experiences," Electron. Notes Theor. Comput. Sci., vol. 185, no. SPEC. ISS., pp. 139-151, Jul. 2007.

[24] A. H. A. Melani, C. A. Murad, A. Caminada Netto, G. F. M. de Souza, and S. I. Nabeta, "Criticality-based maintenance of a coal-fired power plant," Energy, vol. 147, pp. 767-781, Mar. 2018.

[25] R. Ferdous, F. Khan, R. Sadiq, P. Amyotte, and B. Veitch, "Fault and Event Tree Analyses for Process Systems Risk
Analysis: Uncertainty Handling Formulations,” Risk Anal., vol. 31, no. 1, pp. 86-107, Jan. 2011.

[26] W. Vesely and M. Stamatelatos, Fault Tree Handbook with Aerospace Applications. Washington, DC, USA: NASA Office of Safety and Mission Assurance, 2002.

[27] E. Ruijters, D. Guck, P. Drolenga, and M. Stoelinga, "Fault maintenance trees: Reliability centered maintenance via statistical model checking," in 2016 Annual Reliability and Maintainability Symposium (RAMS), 2016, pp. 1-6.

[28] A. Sarkar, S. C. Panja, and D. Das, "Fault tree analysis of Rukhia gas turbine power plant," HKIE Trans., vol. 22, no. 1, pp. 32-56, Jan. 2015.

[29] V. Vasconcelos, M. Senne, and R. O. O. Marques, "Use of Casual Tree Method for Investigation of Incidents and Accidents Involving Radioactive," in International Nuclear Atlantic Conference - INAC 2013, 2013.

[30] K. Brik and F. ben Ammar, "Causal tree analysis of depth degradation of the lead acid battery," Journal of Power Sources, vol. 228, pp. 39-46, 2013.

[31] Y. E. Senol, Y. V. Aydogdu, B. Sahin, and I. Kilic, "Fault Tree Analysis of chemical cargo contamination by using fuzzy approach,” Expert Syst. Appl., vol. 42, no. 12, pp. 5232-5244, Jul. 2015.

[32] P. Guo, Z. Wang, L. Sun, and X. Luo, "Characteristic analysis of the efficiency hill chart of Francis turbine for different water heads," Adv. Mech. Eng., vol. 9, no. 2, pp. 1-8, Feb. 2017. 